\title{
Reliability Analysis of a Two Dissimilar Unit Cold Standby System with Three Modes Using Kolmogorov Forward Equation Method
}

\author{
*1Uba Ahmad Ali, 2Naziru Idris Bala and 2lbrahim Yusuf \\ 1Department of Mathematics, Faculty of Science, Usmanu Danfodiyo University, Sokoto, Nigeria \\ 2Department of Mathematics, Bayero University Kano, Nigeria

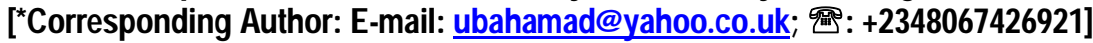

\begin{abstract}
Reliability is an important area that is receiving attention globally. This paper studies reliability analysis of a two-dissimilar-unit cold standby system with three modes using Kolmogorov forward equation's method, in which its reliability and availability analysis are evaluated. This proposed system has been investigated under three modes: normal, partial failure, total failure. The failure and the repair time are assumed to have exponential distribution. The effect of failure rate on meantime to system failure $(M T S F)$, steady state availability $\left(A_{T}(\infty)\right)$ and the profit function $P F(\infty)$ of the system are compared. It was found that $M T S F, A_{T}(\infty)$, and $P F(\infty)$ are decreasing with the increase of the failure rate.

Keywords: Cost analysis, Meantime to System Failure, Steady State Availability and Kolmogorov forward equation.
\end{abstract}

\section{INTRODUCTION}

Reliability is a vital for proper utilization and maintenance of any system. It involves technique for increasing system effectiveness through reducing failure frequency and maintenance cost minimization. Studies on two unit redundant system with repair can be obtained in Arora(1976), Emara and El-said (1992), Goel et al. (1986). Damcese and Temraz (2010), Goel and Gupta (1984) have studied the analysis of two unit parallel system. El-said (2008), El-said and El- sherbeny (2005), Gopalan and Nagawalia (1985), and Rander (1994) have studied the cost analysis with preventive maintenance of different system. El-said and Elsherbeny (2005) by using linear first order differential equation evaluated the reliability and availability characteristics of two different systems. Gupta and Mittal (2006) studied the stochastic behavior of a two unit warm standby system with two types of repairmen and patience time. Mokaddis et al. (2009) using linear first order differential equation evaluated the reliability and availability characteristics of two-dissimilar-unit cold standby system with three mode for which no cost benefit analysis was considered. El-said (1994) contributed on stochastic analysis of a two-dissimilar-unit standby redundant system. This research work deals with the reliability analysis of a two-dissimilarunit cold standby system with three modes using
Kolmogorov forward equation method. Initially one unit is operative and the other unit is kept as a cold standby, i.e. it does not fail while standing by. Each unit works with three modes; normal, partial failure and total failure. The system fails when both units fail totally. The failure and the repair rate are assumed to have exponential distribution. The effect of failure rate on meantime to system failure, steady state availability and profit function are determined based on the assumed numerical values given to the system parameters and reliability measures.

\section{METHODOLOGY}

\section{Assumption and Notations}

\section{Assumptions}

The following assumptions were adopted:

i. The system works with one unit that is operative and the other is kept as a cold standby, i.e. it does not fail while standing by unless it goes into operation.

ii. The standby is switched to operative state in negligible time.

iii. The operative units have three modes of operation: these are normal, O-mode, which means the functioning of the units with full capacity, partial failure, p-mode which means the functioning of the units with reduced capacity at specified level and the 
Ali et al.: Reliability Analysis of a Two Dissimilar Unit Cold Standby System with Three Modes Using Kolmogorov.........

total failure, T-mode which means the capacity goes below a specified level.

iv. A unit, which is replaced or repaired in total failure mode. This assumed to go directly to the normal without passing through the partial failure mode.

v. A unit in the normal mode must pass through the partial failure mode to get in to the total failure mode. In the partial failure mode, a unit is working as good as being repaired.

vi. The system is down when all units are nonoperative.

vii. For constructing the probabilistic structure of the system, it is assumed that the failure and repair times have exponential distributions.

\section{Notations}

$\lambda_{1}$; the failure rate of the $i^{\text {th }}$ unit from $O_{i}$ mode to $P_{i}$-mode, i=1,2.

$\alpha_{i}$; the failure rate of the $i^{\text {th }}$ unit from $P_{i}$ mode to $T_{i}$-mode, $\mathrm{i}=1,2$.

$\mu_{i}$; the repair rate of the $i^{\text {th }}$ unit from $T_{i}$ mode to $O_{i}$-mode, i=1,2.

$\beta_{i}$; the repair rate of the $i^{\text {th }}$ unit from $P_{i}$ mode to $O_{i}$-mode, i=1,2.

$O_{i} ;$ the $i^{\text {th }}$ unit is operative, $\mathrm{i}=1,2$

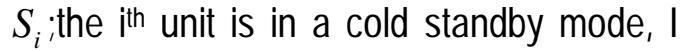

$=1,2$.

$P_{i}$; the $i^{\text {th }}$ unit is partial failure, $\mathrm{i}=1,2$.

$T_{i}$; the $i^{\text {th }}$ unit is in a total failure mode, $\mathrm{i}=1$, 2.

$S_{i}$; denotes the state of the systems i=0 to 8.

Stochastic Behaviour of the System

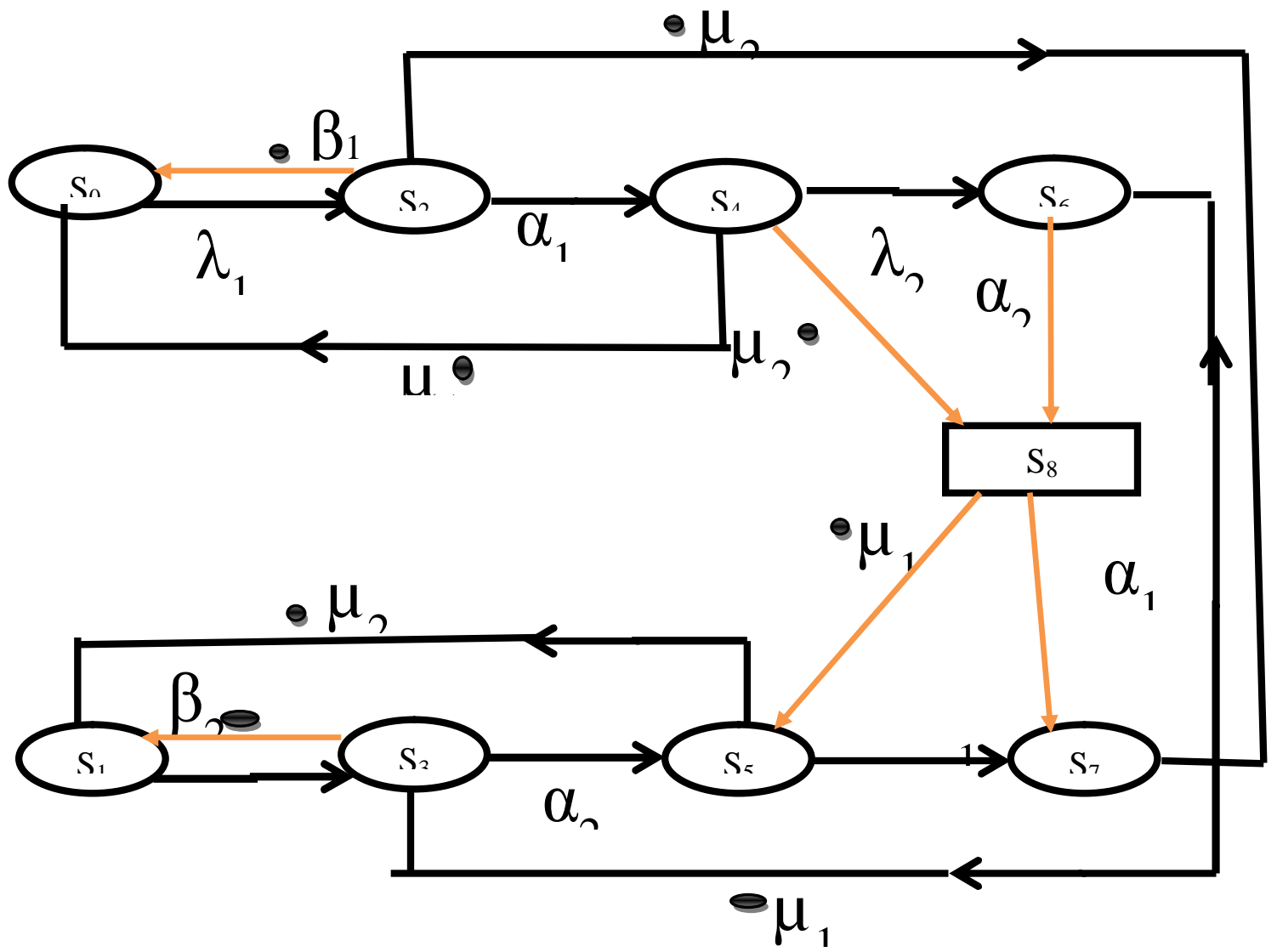

Figure 1: Transition Diagram of the System
$\bigcirc$ Up State
$\square$ Down State
- Regeneration 


\section{States of the System}

state $S_{0}$; $\quad$ unit one is operative, unit two is in a cold standby.

State $S_{1}$; $\quad$ unit two is operative, unit one is in a cold standby.

state $S_{2}$; $\quad$ one unit is in a partial failure, unit two is in a cold standby.

state $S_{3}$; $\quad$ unit one is in a cold standby, two unit is in a partial failure mode.

state $S_{4}$; $\quad$ unit one is in a total failure modes, unit two is operative.

state $S_{5}$; $\quad$ unit one is operative, unit two is in a total failure mode.

state $S_{6}$; $\quad$ unit one is operative, unit two is in a total failure mode.

state $S_{7}$; $\quad$ unit one is in a partial failure mode, unit two is in a total failure mode.

state $S_{8}$; $\quad$ both units are in total failure mode. i.e. the system is down completely.

\section{Mean Time to System Failure}

By using the method of kolmogorov forward equations and the above mentioned set of assumptions, the mean time to system failure MTSF for the proposed system will be evaluated. From Figure 1 , let $P_{i}(t)$ be the probability that the system at time $(\mathrm{t}>=0)$ in state $\mathrm{s}_{\mathrm{i}}$. Let $P(t)$ denote the probability row vector at time t, the initial conditions for this problem are

$$
p(0)=\left[p_{0}(0), p_{1}(0), p_{2}(0), p_{3}(0), p_{4}(0), p_{5}(0), p_{6}(0), p_{7}(0), p_{8}(0)\right]=[1,0,0,0,0,0,0,0,0]
$$

By employing the method of kolmogorov forward equations, El-Said and Elshebeny (2005), the following differential equations are obtained:

$$
\begin{aligned}
& \frac{d p_{0}(t)}{d t}=-\lambda_{1} p_{0}(t)+\beta_{1} p_{2}(t)+\mu_{1} p_{4}(t) \\
& \frac{d p_{1}(t)}{d t}=-\lambda_{2} p_{1}(t)+\beta_{2} p_{3}(t)+\mu_{2} p_{5}(t) \\
& \frac{d p_{2}(t)}{d t}=-\left(\alpha_{1}+\beta_{1}\right) p_{2}(t)+\mu_{2} p_{7}(t)+\lambda_{1} p_{0}(t) \\
& \frac{d p_{3}(t)}{d t}=-\left(\alpha_{2}+\beta_{2}\right) p_{3}(t)+\mu_{1} p_{6}(t)+\lambda_{2} p_{1}(t) \\
& \frac{d p_{4}(t)}{d t}=-\left(\lambda_{2}+\mu_{1}\right) p_{4}(t)+\alpha_{1} p_{2}(t)+\mu_{2} p_{8}(t) \\
& \frac{d p_{5}(t)}{d t}=-\left(\lambda_{1}+\mu_{2}\right) p_{5}(t)+\alpha_{2} p_{3}(t)+\mu_{1} p_{8}(t) \\
& \frac{d p_{6}(t)}{d t}=-\left(\alpha_{2}+\mu_{1}\right) p_{6}(t)+\lambda_{2} p_{4}(t) \\
& \frac{d p_{7}(t)}{d t}=-\left(\alpha_{1}+\mu_{2}\right) p_{7}(t)+\lambda_{1} p_{5}(t) \\
& \frac{d p_{8}(t)}{d t}=-\left(\mu_{1}+\mu_{2}\right) p_{8}(t)+\alpha_{2} p_{6}(t)+\alpha_{1} p_{7}(t)
\end{aligned}
$$

This can be written in the matrix form as

$$
\frac{d p(t)}{d t}=Q^{*} p
$$


Where,

$$
Q=\left(\begin{array}{ccccccccc}
-\lambda_{1} & 0 & \beta_{1} & 0 & \mu_{1} & 0 & 0 & 0 & 0 \\
0 & -\lambda_{2} & 0 & \beta_{2} & 0 & \mu_{2} & 0 & 0 & 0 \\
\lambda_{1} & 0 & -\left(\alpha_{1}+\beta_{1}\right) & 0 & 0 & 0 & 0 & \mu_{2} & 0 \\
0 & \lambda_{2} & 0 & -\left(\alpha_{2}+\beta_{2}\right) & 0 & 0 & \mu_{1} & 0 & 0 \\
0 & 0 & \alpha_{1} & 0 & -\left(\lambda_{2}+\mu_{1}\right) & 0 & 0 & 0 & \mu_{2} \\
0 & 0 & 0 & \alpha_{2} & 0 & -\left(\lambda_{1}+\mu_{2}\right) & 0 & 0 & \mu_{1} \\
0 & 0 & 0 & 0 & \lambda_{2} & 0 & -\left(\alpha_{2}+\mu_{1}\right) & 0 & 0 \\
0 & 0 & 0 & 0 & 0 & \lambda_{1} & 0 & -\left(\alpha_{1}+\mu_{2}\right) & 0 \\
0 & 0 & 0 & 0 & 0 & 0 & \alpha_{2} & \alpha_{1} & -\left(\mu_{1}+\mu_{2}\right)
\end{array}\right)
$$

To calculate the MTSF, we took the transpose of the matrix $\mathrm{Q}$ and deleted the rows and columns for the absorbing state. The new matrix is called $\mathrm{A}$

$$
A=\left[\begin{array}{cccccccc}
-\lambda_{1} & 0 & \lambda_{1} & 0 & 0 & 0 & 0 & 0 \\
0 & -\lambda_{2} & 0 & \lambda_{2} & 0 & 0 & 0 & 0 \\
\beta_{1} & 0 & -\left(\alpha_{1}+\beta_{1}\right) & 0 & \alpha_{1} & 0 & 0 & 0 \\
0 & \beta_{2} & 0 & -\left(\alpha_{2}+\beta_{2}\right) & 0 & \alpha_{2} & 0 & 0 \\
\mu_{1} & 0 & 0 & 0 & -\left(\lambda_{2}+\mu_{1}\right) & 0 & \lambda_{2} & 0 \\
0 & \mu_{2} & 0 & 0 & 0-\left(\lambda_{1}+\mu_{2}\right) & 0 & \lambda_{1} \\
0 & 0 & 0 & \mu_{1} & 0 & 0-\left(\alpha_{2}+\mu_{1}\right) & 0 \\
0 & 0 & \mu_{2} & 0 & 0 & 0 & 0-\left(\alpha_{1}+\mu_{2}\right)
\end{array}\right]
$$

Therefore, the expected time to reach an absorbing state was calculated from

$$
M T S F=E\left[T_{p(0) \rightarrow p(\text { absorbing })}\right]=p(0) *\left(-A^{-1}\right) *\left(\begin{array}{l}
1 \\
1 \\
1 \\
1 \\
1 \\
1 \\
1 \\
1
\end{array}\right)
$$

MTSF $=\left[\mu_{1}^{2} \alpha_{1}^{2}+\mu_{1}^{2} \alpha_{1} \mu_{2}+\mu_{1}^{2} \alpha_{1} \beta_{1}+\mu_{1}^{2} \beta_{1} \mu_{2}+\mu_{1}^{2} \lambda_{2} \alpha_{1}^{2}+\mu_{1} \lambda_{1} \alpha_{1} \beta_{1}+\mu_{1} \lambda_{2} \beta_{1} \mu_{2}+\mu_{1} \alpha_{1}^{2} \alpha_{2}+\mu_{1} \alpha_{1} \alpha_{2} \mu_{2}+\right.$ $\left.\mu_{1} \alpha_{1} \alpha_{2} \beta_{1}+\mu_{1} \beta_{1} \mu_{2} \alpha_{2}+\alpha_{1}{ }^{2} \alpha_{2} \lambda_{2}+\alpha_{2} \mu_{2} \alpha_{1} \lambda_{1}+\lambda_{2} \alpha_{1} \alpha_{2} \beta_{1}+\lambda_{2} \beta_{1} \mu_{2} \alpha_{2}\right] /\left(\lambda_{1} \lambda_{2} \alpha_{1}\right)\left(\alpha_{1} \mu_{1}+\alpha_{1} \alpha_{2}+\alpha_{2} \mu_{2}\right)$ $+\mu_{1}\left[\lambda_{1} \alpha_{1} \beta_{2}+\lambda_{1} \beta_{2} \mu_{2}+\alpha_{2} \mu_{2} \alpha_{1}+\alpha_{1} \beta_{2} \mu_{2}+\beta_{2} \mu_{2}^{2}+\mu_{2}^{2} \alpha_{2}\right] /\left(\lambda_{1} \lambda_{2} \alpha_{2}\right)\left(\alpha_{1} \mu_{1}+\alpha_{1} \alpha_{2}+\alpha_{2} \mu_{2}\right)+$ $\left[\alpha_{1} \mu_{1}^{2}+\mu_{1}^{2} \mu_{2}+\mu_{1} \lambda_{2} \alpha_{1}+\mu_{1} \lambda_{2} \mu_{2}+\alpha_{2} \mu_{1} \alpha_{1}+\mu_{1} \mu_{2} \alpha_{2}+\lambda_{2} \alpha_{1} \alpha_{2}+\mu_{2} \alpha_{2} \lambda_{2}\right] / \lambda_{2} \alpha_{1}\left(\alpha_{1} \mu_{1}+\alpha_{1} \alpha_{2}+\alpha_{2} \mu_{2}\right)+$ $\mu_{1}\left[\lambda_{1} \alpha_{1}+\lambda_{1} \mu_{2}+\mu_{2} \alpha_{1}+\mu_{2}^{2}\right] / \lambda_{1} \alpha_{2}\left(\alpha_{1} \mu_{1}+\alpha_{1} \alpha_{2}+\alpha_{2} \mu_{2}\right)+\left(\alpha_{1} \mu_{1}+\mu_{2} \mu_{1}+\alpha_{1} \alpha_{2}+\alpha_{2} \mu_{2}\right) / \lambda_{2}\left(\alpha_{1} \mu_{1}+\alpha_{1} \alpha_{2}+\alpha_{2} \mu_{2}\right)$ $\mu_{1}\left[\lambda_{1}+\mu_{2}\right] / \lambda_{1}\left(\alpha_{1} \mu_{1}+\alpha_{1} \alpha_{2}+\alpha_{2} \mu_{2}\right)+\left(\alpha_{1}+\mu_{2}\right) /\left(\alpha_{1} \mu_{1}+\alpha_{1} \alpha_{2}+\alpha_{2} \mu_{2}\right)+\mu_{1} /\left(\alpha_{1} \mu_{1}+\alpha_{1} \alpha_{2}+\alpha_{2} \mu_{2}\right)$

Multiplying each term by the L.C.M. $\left(\alpha_{1} \mu_{1}+\alpha_{1} \alpha_{2}+\alpha_{2} \mu_{2}\right)$ and simplifying the terms, the following equations were obtained: 


$$
\begin{aligned}
& \operatorname{MTSF}=\left(z_{1}+z_{2}+z_{3}+z_{4}\right) / z_{5} \\
& z_{1}=\alpha_{2}\left(\alpha_{1}+\mu_{2}\right)\left(\alpha_{2}+\mu_{1}\right)\left(\alpha_{1}+\beta_{2}\right)\left(\lambda_{2}+\mu_{1}\right) \\
& z_{2}=\alpha_{1} \mu_{2}\left(\alpha_{1}+\mu_{2}\right)\left(\lambda_{1}+\mu_{2}\right)\left(\lambda_{2}+\alpha_{2}+\beta_{2}\right) \\
& z_{3}=\lambda_{1} \alpha_{2}\left(\alpha_{1}+\mu_{2}\right)\left[\left(\lambda_{2}+\mu_{1}\right)\left(\alpha_{2}+\mu_{1}\right)+\alpha_{1}\left(\lambda_{2}+\alpha_{2}\right)\right. \\
& z_{4}=\alpha_{1} \alpha_{2} \mu_{1} \lambda_{1}\left(\alpha_{1}+\lambda_{1}\right) \\
& z_{5}=\alpha_{1} \alpha_{2} \lambda_{1} \lambda_{2}\left(\alpha_{1} \mu_{1}+\alpha_{1} \alpha_{2}+\alpha_{2} \mu_{2}\right)
\end{aligned}
$$

\section{Availability Analysis for the System}

For the availability analysis of the states of the system represented in Figure 1, the initial conditions for this problem are the same as for the reliability case:

$$
p(0)=\left[p_{0}(0), p_{1}(0), p_{2}(0), p_{3}(0), p_{4}(0), p_{5}(0), p_{6}(0), p_{7}(0), p_{8}(0)\right]=[1,0,0,0,0,0,0,0,0]
$$

From the equation (3), the steady state availability could be obtained using the following procedure. In the steady state situation, the derivatives of the state probabilities become zero (El-Said and Elshebeny, 2005). That allowed to calculate the steady state availability for the system defined as:

$A(\propto)=1-p_{8}(\propto)$

we also have equation (9) been satisfied:

$Q * p(\propto)=0$

Thus, to obtain $P_{8}(\infty)$, equation (7) was solved under the following normalization condition

$$
\sum_{i=1}^{8} p_{i}(\propto)=1
$$

This was done by substituting equation (8) in any of the redundant rows in equation (3) to yield equation (11).

$$
B=\left[\begin{array}{lllllllll}
\lambda_{1} & 0 & \beta_{1} & 0 & \mu_{1} & 0 & 0 & 0 & 0 \\
0 & -\lambda_{2} & 0 & \beta_{2} & 0 & \mu_{2} & 0 & 0 & 0 \\
\lambda_{1} & 0 & \left(\alpha_{1}+\beta_{1}\right) & 0 & 0 & 0 & 0 & \mu_{2} & 0 \\
0 & \lambda_{2} & 0 & -\left(\alpha_{2}+\beta_{2}\right) & 0 & 0 & \mu_{1} & 0 & 0 \\
0 & 0 & \alpha_{1} & 0 & -\left(\lambda_{2}+\mu_{1}\right) & 0 & 0 & 0 & \mu_{2} \\
0 & 0 & 0 & \alpha_{2} & 0-\left(\lambda_{1}+\mu_{2}\right) & 0 & 0 & \mu_{1} \\
0 & 0 & 0 & 0 & \lambda_{2} & 0-\left(\alpha_{2}+\mu_{1}\right) & 0 & 0 \\
0 & 0 & 0 & 0 & 0 & \lambda_{1} & 0-\left(\alpha_{1}+\mu_{2}\right) & 0 \\
1 & 1 & 1 & 1 & 1 & 1 & 1 & 1 & 1
\end{array}\right]
$$

$$
B \times\left[\begin{array}{c}
p_{0}(\infty) \\
p_{1}(\infty) \\
p_{2}(\infty) \\
p_{3}(\infty) \\
p_{4}(\infty) \\
p_{5}(\infty) \\
p_{6}(\infty) \\
p_{7}(\infty) \\
p_{8}(\infty)
\end{array}\right]=\left[\begin{array}{l}
0 \\
0 \\
0 \\
0 \\
0 \\
0 \\
0 \\
0 \\
1
\end{array}\right]
$$


For Figure 1, the steady state availability of the proposed system $A_{T}(\infty)$ is given by:

$A_{T}(\infty)={ }_{1}-a_{1} a_{2} \lambda_{2} \lambda_{1}\left(\mu_{1} a_{1}+a_{2} a_{1}+\mu_{2} a_{2}\right) /\left(\mu_{1}{ }^{3} a_{1} a_{2} \mu_{2}+\lambda_{1} a_{1} \mu_{2}{ }^{2} \lambda_{2} a_{2}+{ }_{2} \lambda_{1} a_{1} \lambda_{2} a_{2}{ }^{2} \mu_{2} \mu_{1}+\lambda_{1} a_{1} \lambda_{2} a_{2}{ }^{2} \mu_{2}+\right.$ $\mu_{1} \lambda_{1} \lambda_{2} a_{2} a_{1}^{2}+\mu_{2} \lambda_{2} a_{2} \lambda_{1} \mu_{1}^{2}+\mu_{1} \lambda_{1} \mu_{2} \lambda_{2} a_{2}^{2}+\lambda_{1} \lambda_{2} a_{2}^{2} a_{1}^{2}+\lambda_{1} \lambda_{2} a_{2} a_{1}^{2} \mu_{2}+\mu_{1} \lambda_{2} a_{2}^{2} a_{1}^{2}+\mu_{1} \lambda_{2} \beta_{1} \mu_{2} a_{2}^{2}+\mu_{1}$ $\lambda_{2} \mu_{2} \alpha_{2}^{2} a_{1}+\mu_{1} \lambda_{2} \mu_{2}^{2} \beta_{1} a_{2}+\mu_{1} a_{2}^{2} \mu_{2} a_{1} \beta_{1}+\mu_{1} \mu_{2}^{2} a_{2}^{2} \beta_{1}+\mu_{1} \lambda_{1} \lambda_{2} \mu_{2}^{2} a_{2}+\mu_{1} \lambda_{1} \mu_{2}^{2} a_{2}^{2}+\mu_{1} \lambda_{1} \lambda_{2} \mu_{2} a_{1}^{2}+\mu_{1}$ $\lambda_{1} \mu_{2}^{2} a_{1} \lambda_{2}+\mu_{1} \lambda_{2} a_{1}^{2} \mu_{2}^{2}+\mu_{1} \lambda_{2} a_{1} \mu_{2}{ }^{3}+\mu_{1} \lambda_{1} a_{2} a_{1}^{2} \mu_{2}+\mu_{1} \lambda_{1} a_{1}^{2} \mu_{2} \beta_{2}+\mu_{1} \lambda_{1} a_{2} a_{1} \mu_{2}^{2}+\mu_{1} \lambda_{1} \mu_{2}^{2} a_{1} \beta_{2}+{ }_{2} \mu_{1}$ $\mu_{2} a_{2}^{2} a_{1}^{2}+\mu_{1} a_{1}^{2} \beta_{2} \mu_{2} a_{2}+\mu_{1} \mu_{2}^{2} a_{1}^{2} a_{2}+\mu_{1} a_{1}^{2} \mu_{2}^{2} \beta_{2}+2 \mu_{1} \mu_{2}^{2} a_{1} a_{2}^{2}+\mu_{1} \mu_{2}{ }^{3} a_{1} a_{2}+\mu_{1} a_{1} \mu_{2}^{2} \beta_{2} a_{2}+\mu_{1}$ $a_{1} \mu_{2}{ }^{3} \beta_{2}+\lambda_{1} \mu_{2} a_{2}^{2} a_{1}^{2}+\lambda_{1} \mu_{2}^{2} a_{1} a_{2}^{2}+\mu_{1}{ }^{3} \lambda_{1} \mu_{2} a+\mu_{1}{ }^{3} \mu_{2} a_{2} \beta_{1}+\mu_{1}{ }^{2} \mu_{2} a_{1} \lambda_{2} \lambda_{1}+{ }_{2} \mu_{1}{ }^{2} \mu_{2} a_{2} a_{1} \lambda_{1}+\mu_{1}{ }^{2} \mu_{2}$ $a_{1} \beta_{2} \lambda_{1}+\mu_{1}^{2} \lambda_{1} \mu_{2}^{2} a_{2}+\mu_{1}^{2} \lambda_{1} \mu_{2} a_{2}^{2}+\mu_{1} a_{1}^{2} \lambda_{2} a_{2}+\mu_{1}{ }^{2} \lambda_{2} \mu_{2} a_{1}^{2}+\mu_{1}^{2} \lambda_{2} a_{2} \mu_{2} a_{1}+\mu_{1}^{2} \mu_{2}^{2} a_{1} \lambda_{2}+\mu_{1}^{2} \lambda_{2} a_{2}$ $\beta_{1} \mu_{2}+{ }_{2} \mu_{1}{ }^{2} \alpha_{2} a_{1}{ }^{2} \mu_{2}+\mu_{1}{ }^{2} a_{1}{ }^{2} \mu_{2} \beta_{2}+\mu_{1}{ }^{2} a_{1} a_{2}{ }^{2} \mu_{2}+\mu_{1}{ }^{2} a_{1} \mu_{2} \beta_{1} a_{2}+2 \mu_{1}{ }^{2} a_{1} \mu_{2}{ }^{2} a_{2}+\mu_{1}{ }^{2} a_{1} \mu_{2}{ }^{2} \beta_{2}+\mu_{1}{ }^{2} \beta_{1}$ $\left.\mu_{2} a_{2}^{2}+\mu_{1} \mu_{2}{ }^{2} \beta_{1} a_{2}+{ }_{2} \mu_{1} \lambda_{1} \mu_{2} a_{2}{ }^{2} a_{1}+{ }_{2} \lambda_{2} a_{1}{ }^{2} a_{2} \mu_{1} \mu_{2}+\lambda_{2} \lambda_{1} a_{2}{ }^{2} \mu_{1} a_{1}+\lambda_{2} a_{1}{ }^{2} a_{2} \mu_{1} \lambda_{1}+{ }_{2} \lambda_{2} a_{1} \mu_{2} \mu_{1} a_{2}{ }^{2}\right)$ (12)

$$
\begin{aligned}
& \text { Where } N=\lambda_{1} \lambda_{2} h_{5} \\
& D=\lambda_{1} \lambda_{2} \alpha_{1} \mu_{1}\left(h_{1}+\mu_{2} h_{2}\right)+\lambda_{1} \lambda_{2} \mu_{1}\left(h_{4}+\alpha_{1} \mu_{1} h_{3}\right)+\lambda_{1}{ }^{2} \lambda_{2}{ }^{2} \alpha_{1} \alpha_{2} h \\
& h_{1}=\left(\alpha_{1}+\mu_{2}\right)\left[\mu_{1}^{2}\left(\lambda_{2}+\beta_{2}\right)+\alpha_{2}^{2}\left(\lambda_{1}+2 \mu_{1}\right)+\lambda_{2} \mu_{1}\left(\lambda_{1}+2 \alpha_{2}\right)\right] \\
& h_{2}=\left(\alpha_{2}+\beta_{2}\right)\left(\lambda_{1} \alpha_{1}+\lambda_{1} \mu_{2}+\alpha_{1} \mu_{2}\right)+\left(\lambda_{1} \mu_{1}+\mu_{2}^{2}\right)+\alpha_{2}\left(\alpha_{2} \beta_{1}+\mu_{2} \beta_{2}+\alpha 2 \beta_{2}\right)+2 \mu_{1} \alpha_{2}\left(\alpha_{2}+\beta_{1}\right) \\
& h_{3}=\left(\alpha_{2}+\mu_{2}\right)\left(\lambda_{1} \mu_{1}+\lambda_{1} \lambda_{2}+2 \mu_{1} \alpha_{2}\right)+\mu_{1}\left(\mu_{1}+\lambda_{2}\right)+\left(\lambda_{1}+\alpha_{1}+\beta_{1}\right)+\mu_{2}\left(\alpha_{1} \mu_{2}+\alpha_{2} \beta_{1}+\lambda_{1} \alpha_{2}\right) \\
& h_{4}=\alpha_{1} \alpha_{2}\left(\mu_{1}+\alpha_{2}\right)\left(2 \lambda_{1} \mu_{2}+\lambda_{2} \alpha_{1}\right)+\lambda_{2} \mu_{1}\left[\mu_{2}\left(\alpha_{1}^{2}+\alpha_{2} \beta_{1}\right)+\alpha_{2}^{2}\left(\alpha_{1}+\beta_{1}\right)\right] \\
& h_{5}=\left(\mu_{1}+\mu_{2}\right)\left(\alpha_{1}+\alpha_{2}+\mu_{1}+\mu_{2}\right)
\end{aligned}
$$

The solution of equation (12) above produced the steady state probabilities of the availability analysis.

\subsection{Busy Period Analysis $B P(\infty)$}

The initial conditions for this problem are the same as for the reliability case:

$$
\begin{aligned}
& p(0)=\left[p_{0}(0), p_{1}(0), p_{2}(0), p_{3}(0), p_{4}(0), p_{5}(0), p_{6}(0), p_{7}(0), p_{8}(0)\right] \\
& =[1,0,0,0,0,0,0,0,0]
\end{aligned}
$$

The differential equation form can be expressed as shown in equation (13).

$$
D=\left[\begin{array}{lllllllll}
\lambda_{1} & 0 & \beta_{1} & 0 & \mu_{1} & 0 & 0 & 0 & 0 \\
0 & -\lambda_{2} & 0 & \beta_{2} & 0 & \mu_{2} & 0 & 0 & 0 \\
\lambda_{1} & 0 & \left(\alpha_{1}+\beta_{1}\right) & 0 & 0 & 0 & 0 & \mu_{2} & 0 \\
0 & \lambda_{2} & 0 & -\left(\alpha_{2}+\beta_{2}\right) & 0 & 0 & \mu_{1} & 0 & 0 \\
0 & 0 & \alpha_{1} & 0 & -\left(\lambda_{2}+\mu_{1}\right) & 0 & 0 & 0 & \mu_{2} \\
0 & 0 & 0 & \alpha & 0 & -\left(\lambda_{1}+\mu_{2}\right) & 0 & 0 & \mu_{1} \\
0 & 0 & 0 & 0 & \lambda_{2} & 0 & -\left(\alpha_{2}+\mu_{1}\right) & 0 & 0 \\
0 & 0 & 0 & 0 & 0 & \lambda_{1} & 0 & -\left(\alpha_{1}+\mu_{2}\right) & 0 \\
1 & 1 & 1 & 1 & 1 & 1 & 1 & 1 & 1
\end{array}\right]
$$




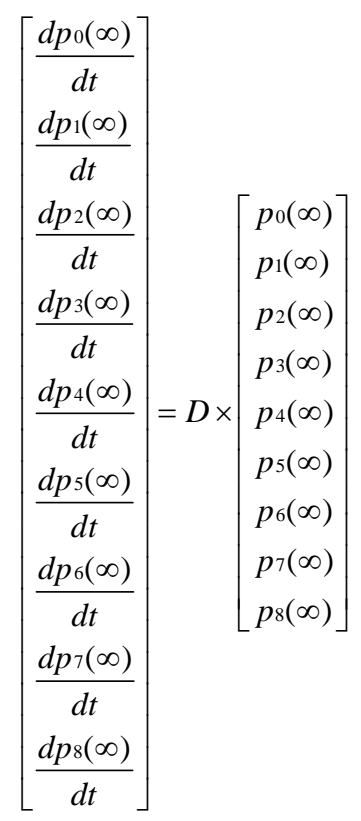

The steady state busy period was obtained using the following procedure. In the steady state, the derivatives of the state probabilities become zero (El-Said and Elshebeny, 2005). That allows us to calculate the steady state probabilities with

$$
\begin{gathered}
B(\propto)=1-\left[p(\propto)+p_{1}(\propto)\right] \\
Q * p(\propto)=0
\end{gathered}
$$

The matrix form of equation (15) is given in equation (16)

$$
\begin{aligned}
& Q=\left[\begin{array}{lllllllll}
\lambda_{1} & 0 & \beta_{1} & 0 & 0 & 0 & 0 & 0 & 0 \\
0 & -\lambda_{2} & 0 & \beta_{2} & 0 & \mu_{2} & 0 & 0 & 0 \\
\lambda_{1} & 0 & \left(\alpha_{1}+\beta_{1}\right) & 0 & 0 & 0 & 0 & \mu_{2} & 0 \\
0 & \lambda_{2} & 0 & -\left(\alpha_{2}+\beta_{2}\right) & 0 & 0 & \mu_{1} & 0 & 0 \\
0 & 0 & \alpha_{1} & 0 & -\left(\lambda_{2}+\mu_{1}\right) & 0 & 0 & 0 & \mu_{2} \\
0 & 0 & 0 & \alpha_{2} & 0 & -\left(\lambda_{1}+\mu_{2}\right) & 0 & 0 & \mu_{1} \\
0 & 0 & 0 & 0 & \lambda_{2} & 0 & -\left(\alpha_{2}+\mu_{1}\right) & 0 & 0 \\
0 & 0 & 0 & 0 & 0 & \lambda_{1} & 0 & -\left(\alpha_{1}+\mu_{2}\right) & 0 \\
0 & 0 & 0 & 0 & 0 & 0 & 0 & 0 & -\left(\mu_{1}+\mu_{2}\right)
\end{array}\right] \\
& Q \times\left[\begin{array}{l}
p_{0}(\infty) \\
p_{1}(\infty) \\
p_{2}(\infty) \\
p_{3}(\infty) \\
p_{4}(\infty) \\
p_{5}(\infty) \\
p_{6}(\infty) \\
p_{7}(\infty) \\
p_{8}(\infty)
\end{array}\right]=\left[\begin{array}{l}
0 \\
0 \\
0 \\
0 \\
0 \\
0 \\
0 \\
0 \\
1
\end{array}\right]
\end{aligned}
$$


To obtain $P_{0}(\infty), P_{1}(\infty)$, equation (16) was solved and the following normalization condition. Equation (16) was substituted in any one of the redundant rows in equation (15) to yield equation (17):

$$
D \times\left[\begin{array}{c}
p_{0}(\infty) \\
p_{1}(\infty) \\
p_{2}(\infty) \\
p_{3}(\infty) \\
p_{4}(\infty) \\
p_{5}(\infty) \\
p_{6}(\infty) \\
p_{7}(\infty) \\
p_{8}(\infty)
\end{array}\right]=\left[\begin{array}{l}
0 \\
0 \\
0 \\
0 \\
0 \\
0 \\
0 \\
0 \\
1
\end{array}\right]
$$

The steady state busy period $B P(\infty)$ is given

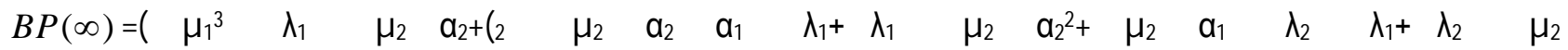
$a_{1}^{2}+\lambda_{1} \quad \mu_{2}^{2} \quad a_{2}+\mu_{2}^{2} \quad a_{1} \quad \lambda_{2}+a_{1}^{2} \quad \lambda_{2} \quad a_{2}+\mu_{2} \quad \lambda_{2} \quad a_{2} \quad \lambda_{1}+\lambda_{2} \quad a_{2} \quad \mu_{2} \quad a_{1}+\lambda_{2} a_{1} a_{2}$

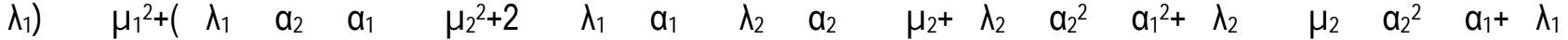

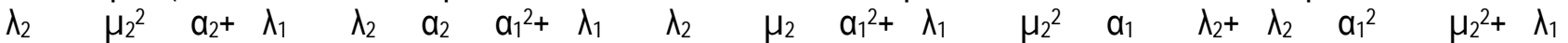

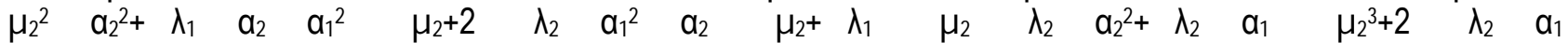
$\begin{array}{lllllllllllllllllll}\mu_{2}{ }^{2} & a_{2}+2 & \lambda_{1} & \mu_{2} & a_{2}{ }^{2} & a_{1}+ & \lambda_{2} & \lambda_{1} & a_{2}{ }^{2} & \left.a_{1}\right) & \mu_{1}+ & \lambda_{1} & a_{1} & \mu_{2}{ }^{2} & \lambda_{2} & a_{2}+ & \lambda_{1} & \mu_{2} & a_{2}{ }^{2}\end{array}$

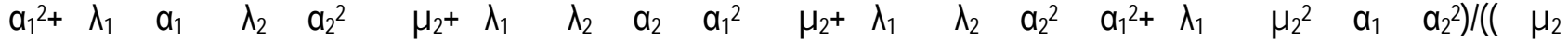

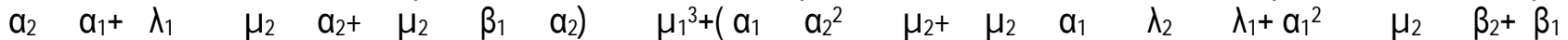

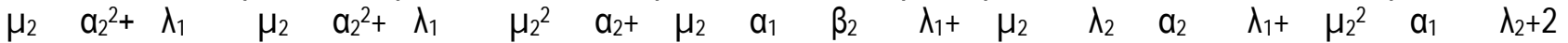

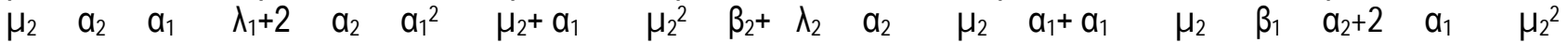

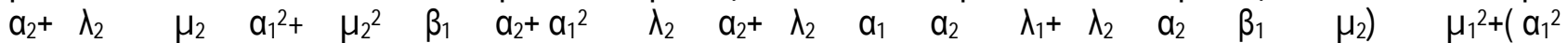

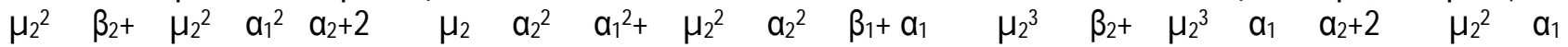

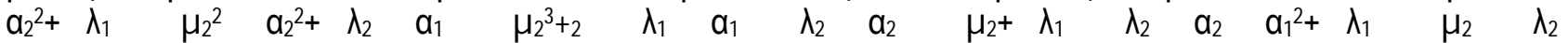
$a_{2}^{2}+\lambda_{2} \quad \mu_{2} \quad a_{2}^{2} \quad a_{1}+\lambda_{1} \quad \lambda_{2} \quad \mu_{2}^{2} \quad a_{2}+\lambda_{1} \quad \lambda_{2} \quad \mu_{2} a_{1}^{2}+\lambda_{1} \quad \mu_{2}^{2} \quad a_{1} \quad \lambda_{2}+\lambda_{1} a_{2} a_{1}^{2}$ $\begin{array}{llllllllllllllllll}\mu_{2}+ & \lambda_{1} & a_{2} & a_{1} & \mu_{2}^{2}+2 & \lambda_{1} & \mu_{2} & a_{2}^{2} & a_{1}+2 & \lambda_{2} & a_{1}{ }^{2} & a_{2} & \mu_{2}+\lambda_{2} & \lambda_{1} & a_{2}^{2} & a_{1}+2 & \lambda_{2} & a_{1}\end{array}$ $\begin{array}{lllllllllllllll}\mu_{2}^{2} & a_{2}+\lambda_{2} & a_{2}^{2} & a_{1}^{2}+\lambda_{2} & a_{1}^{2} & \mu_{2}^{2}+\lambda_{2} & \beta_{1} & \mu_{2} & a_{2}^{2}+\lambda_{2} & \mu_{2}^{2} & \beta_{1} & a_{2}+a_{2}^{2} & \mu_{2} & a_{1} & \beta_{1}+\end{array}$

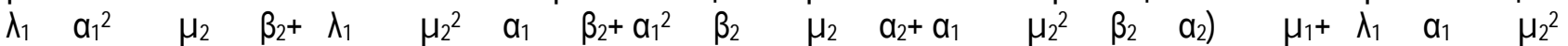
$\begin{array}{llllllllllllllllllllllllll}\lambda_{2} & a_{2}+ & \lambda_{1} & \mu_{2} & a_{2}^{2} & a_{1}^{2}+ & \lambda_{1} & a_{1} & \lambda_{2} & a_{2}^{2} & \mu_{2}+ & \lambda_{1} & \lambda_{2} & a_{2} & a_{1}^{2} & \mu_{2}+ & \lambda_{1} & \lambda_{2} & a_{2}^{2} & a_{1}{ }^{2}+ & \lambda_{1}\end{array}$ $\left.\begin{array}{lll}\mu_{2}^{2} & a_{1} & a_{2}^{2}\end{array}\right)$

\section{RESULTS AND DISCUSSIONS}

Profit Analysis

The expected total profit per unit time incurred to the system in the state is given by

Profit $=$ total revenue - total cost

$$
P F(\infty)=C_{0} A(\infty)-C_{1} B(\infty)
$$

Where,

$P F(\infty)$ : is the profit incurred to the system

$C_{0}$;is the revenue per unit up-time of the system

$C_{1}$; is the cost per unit time which the system is under repaired.
Table 1: Relation Between Failure Rate, MTSF, $A_{T}(\infty)$ and $P F(\infty)$ of the System.

\begin{tabular}{cccc}
\hline$\lambda_{1}$ & MTSF & SAV & Profit \\
\hline 0.10 & 49.6253 & 0.5175 & 431.8572 \\
0.20 & 30.2215 & 0.4260 & 334.5789 \\
0.30 & 23.7535 & 0.3873 & 293.3997 \\
0.40 & 20.5196 & 0.3659 & 270.6531 \\
0.50 & 18.5792 & 0.3523 & 256.2262 \\
0.60 & 17.2856 & 0.3429 & 246.2603 \\
0.70 & 16.3616 & 0.3361 & 238.9633 \\
0.80 & 15.6686 & 0.3308 & 233.3898 \\
0.90 & 15.1296 & 0.3267 & 228.9935 \\
1.00 & 14.6984 & 0.3234 & 225.4372 \\
\hline
\end{tabular}


The failure rate of the system, mean to system failure, system availability, and the profit incurred by the system are summarized in Table 1. Relationship between the system failure rate and the profit incurred is shown in Figure 2. System availability is plotted against its failure in Figure 3 while mean time to system failure is plotted against the system failure rate as shown in Figure 4 . In each of the three cases, it is found that as the system failure increases the dependent variable decreases exponentially.

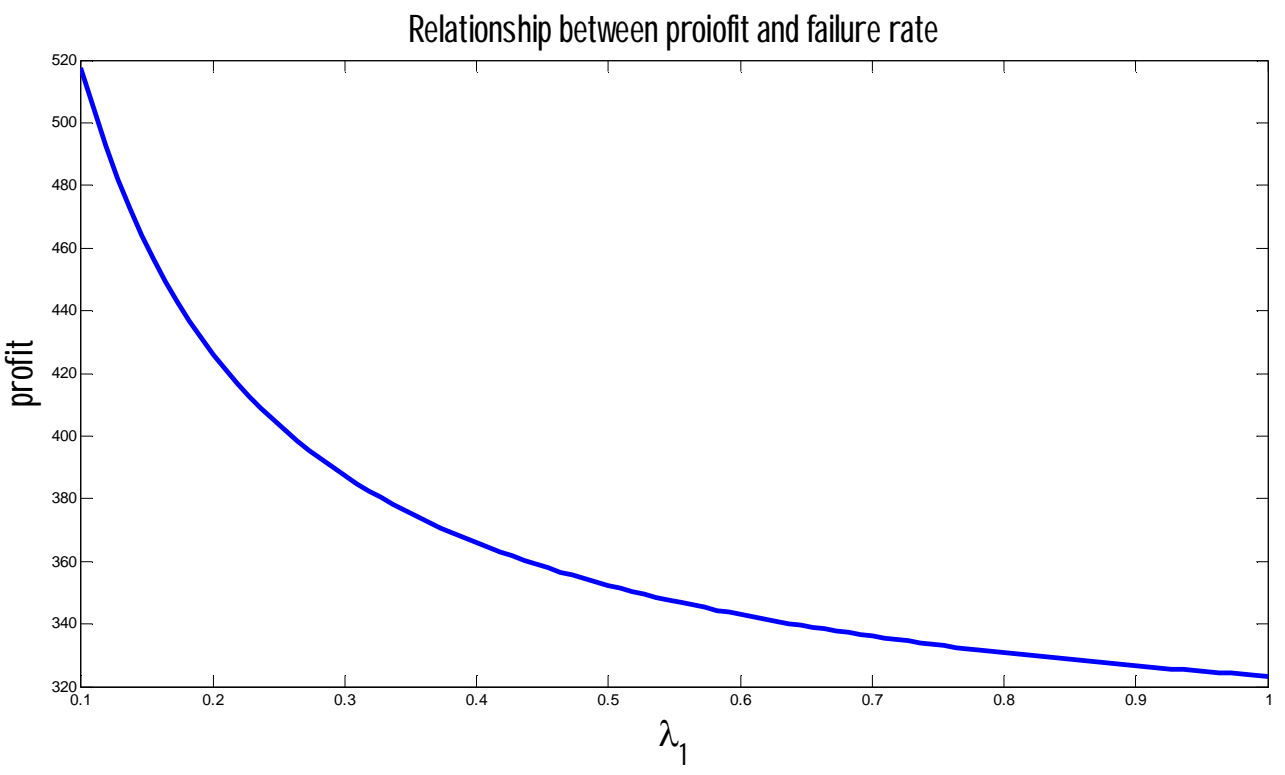

Figure 1: Relationship between $P F(\infty)$ and $\lambda_{1}$

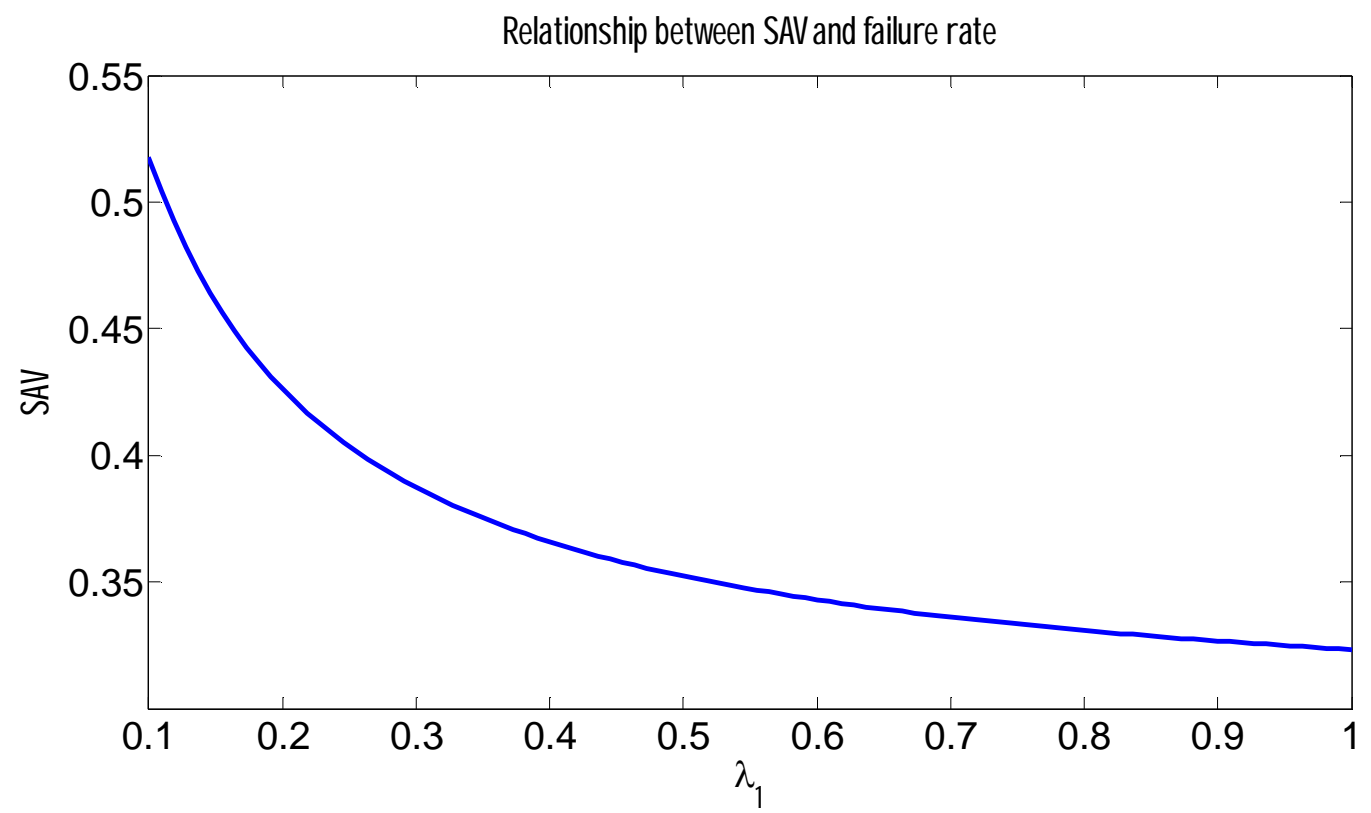

Figure 2: Relationship Between $A_{T}(\infty)$ and $\lambda_{1}$ 


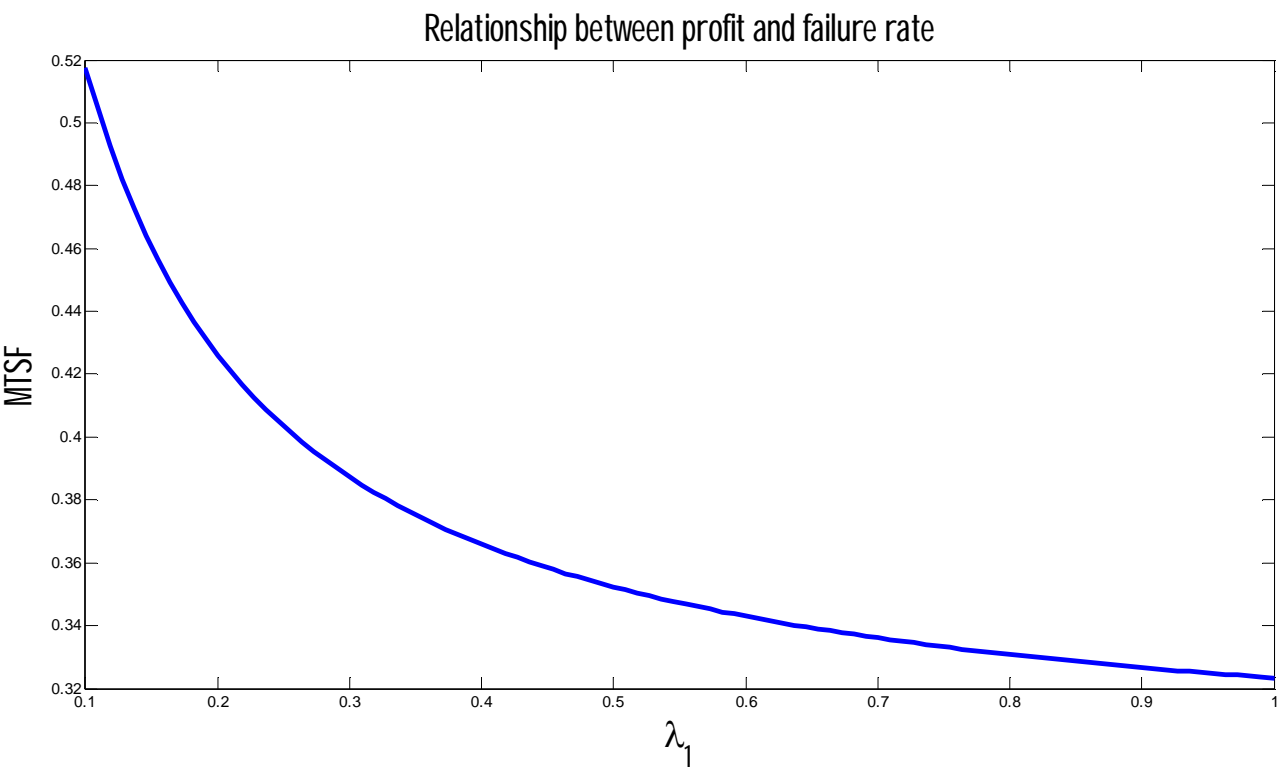

Figure 3: Relationship Between MTSF and $\lambda_{1}$

\section{CONCLUSION}

The characteristics, $M T S F, A_{T}(\infty)$ and $P F(\infty)$ were compared with respect to failure rate $\lambda_{1}$ for the system at fixed values of $\alpha_{1}, \alpha_{2}, \beta_{1}, \beta_{2}, \lambda_{2}, \mu_{1}, \mu_{2}, R_{1}, R_{2}$. It was found that $M T S F, A_{T}(\infty)$ and $P F(\infty)$ are decreasing with the increase of the failure rate $\lambda_{1}$. Periodic inspection, maintenance, and repairs of the units would fetch more profit to the system.

\section{REFERENCES}

Arora, J.A. (1976). Reliability of 2-unit Standby Redundant System with Constrained Repair Time, IEEE Transition, 25(3): 203-205.

Damcese, M.A. and Temraz, N.S. (2010). Analysis of Availability for Reliability Parallel Systems with Different Rates, Asian Journal of Information Technology, 9(4): 231-237.

El-said, K.H.M. (2008). Cost Analysis of a System with Preventive Maintenance by using the Kolmogorov Forward Equation Method. American Journal of Applied Sciences, 5(4):405-410, 2008.

El-said, K.H.M. and El-sherbany M.S. (2005). Evaluation of Reliability and Availability Characteristics of two Difference Systems by using Linear Differential Equations, Journal Mathematics and Statistics, 1: 119-123.

El-said, K.H.M. (1994). Stochastic Analysis of a Twodissimilar- unit Standby Redundant System with Three Modes, Annual Conference Proceeding, ISSR, Cairo University, 29(11): 17-19
El-said, K.H.M. and El-sherbany M.S. (2005). Profit Analysis of a two Unit Cold Standby System with Preventive Maintenance and Random Change in the Units, Journal of Mathematics and Statistics, 1: 71-77.

Emara, S.A.A. and El-said, K.H.M. (1992). Repairable System with Different Failure Modes, Mathematics Conference Proceeding of Non-linear Analysis, Tampa, Florida, USA, Aug.: 19-26.

Goel, L.R. Sharma, G.C. and Gupta, P. (1986). Reliability Analysis of a System with Preventive Inspection and Two Types of Repair, Microelectron. Reliability, 26: 429-433.

Goel, L.R. and Gupta, P. (1984). Stochastic Analysis of a Two-unit Parallel System with Partial and Catastrophic Failure and Preventive Maintenance, Microelectronics Reliability, 24: 881-883.

Gopalan, M.N. and Nagawalia, H.E. (1985). Cost Benefit Analysis of a One Server Two-Unit Cold Standby System with Repair and Preventive Maintenance. Microelectronics Reliability, 25: 267-269.

Gupta, R. and Mittal, M. (2006). Stochastic Analysis of a Compound Redundant System involving Human Failure, Journal of mathematics and Statistics, 2(3): 407-413.

Mokaddis, G.S., El-sherbeny, M.S. and Ayid M.Y. (2009). Stochastic Behaviors of a Two Unit Warm Standby System with Two Types of Repairmen and Patience Time, Journal of Mathematics and Statistics 5(1): 4246.

Rander, M.C. (1994). Cost Analysis of Two dissimilar Cold standby System with Preventive Maintenance and Replacement of Standby, Microelectronics Reliability, 34: 171-174. 